\title{
Ultrasound Ulnar Nerve Measurement in a Healthy Population
}

\author{
Hoel Letissier (D) · Guillaume Dardenne · Alain Saraux • \\ Dominique Le Nen · Bhushan Borotikar · Sandrine Jousse-Joulin
}

Received: November 23, 2020 / Accepted: January 20, 2021 / Published online: February 4, 2021

(C) The Author(s) 2021

\begin{abstract}
Introduction: Ulnar tunnel syndrome at the elbow is a common pathology. The ultrasound cross-sectional area is a well-known metric widely accepted in radiology for the description of nerve entrapment. However, the pathological cut-off value remains challenging. The objectives of this study were to (1) describe the ultrasound cross-sectional area measurement of the ulnar nerve at three locations, and (2) to evaluate the inter-observer reliability by two independent ultrasonographers.
\end{abstract}

H. Letissier $(\bowtie) \cdot$ D. Le Nen

Service de Chirurgie Orthopédique et

Traumatologique, Hôpital de la Cavale Blanche, Boulevard Tanguy Prigent, 29200 Brest, France e-mail: hoel.letissier@chu-brest.fr

H. Letissier - G. Dardenne - B. Borotikar LaTIM, INSERM, UMR 1101, SFR IBSAM, Avenue Foch, 29200 Brest, France

H. Letissier - G. Dardenne - A. Saraux - D. Le Nen · B. Borotikar

Université de Bretagne Occidentale, UBO, 29200

Brest, France

B. Borotikar

Centre Hospitalier Régional Universitaire de Brest, Avenue Foch, 29200 Brest, France

\section{A. Saraux $\cdot$ S. Jousse-Joulin}

Service de Rhumatologie, Hôpital de la Cavale Blanche, Univ Brest, Inserm, LBAI, UMR1227, Brest, France
Methods: One-hundred ulnar nerves of 50 asymptomatic individuals were scanned using B-mode and power Doppler ultrasonography. The ultrasound cross-sectional area measurements of the ulnar nerve were performed at three different levels: $2 \mathrm{~cm}$ proximal to the epicondyle, at the level of the epicondyle, and $2 \mathrm{~cm}$ distal to the epicondyle.

Results: In our healthy population, we found 21,24 and $7 \%$ of ultrasound cross-sectional area ulnar nerve $>8 \mathrm{~mm}^{2}$, respectively, at three different levels of measurement and 4, 7, and $0 \%$ US-CSA ulnar nerve $>10 \mathrm{~mm}^{2}$. The intraclass correlation coefficient measured at three different site levels were good $(0.7943,0.7509)$ to moderate (0.5701).

Conclusions: Almost one-quarter of our healthy population had an ultrasound cross-sectional area ulnar nerve more than $8 \mathrm{~mm}^{2}$ and few more than $10 \mathrm{~mm}^{2}$. A cut-off of ultrasound cross-sectional area ulnar nerve measurement more than $10 \mathrm{~mm}^{2}$ could be considered as pathological. No abnormal elbow ulnar nerve vascularization has been seen. This is the first step towards normal B-mode ulnar nerve values at the elbow to further detect pathological US findings as ulnar nerve entrapment.

Keywords: Cross-sectional area; Elbow; Ulnar nerve; Ulnar nerve entrapment; Ulnar tunnel syndrome; Ultrasonography 


\section{Key Summary Points}

In ulnar nerve entrapment, the ultrasound cross-sectional area is commonly used.

There is no consensus for pathological cutoff value between 8 and $10 \mathrm{~mm}^{2}$.

Almost one-quarter of our healthy population had their ulnar nerve more than $8 \mathrm{~mm}^{2}$.

Only a few of them had an ulnar nerve more than $10 \mathrm{~mm}^{2}$.

A cut-off of more than $10 \mathrm{~mm}^{2}$ could be considered as pathological.

\section{DIGITAL FEATURES}

This article is published with digital features, including a summary slide, to facilitate understanding of the article. To view digital features for this article go to https://doi.org/10.6084/ m9.figshare.13607321.

\section{INTRODUCTION}

The ulnar nerve entrapment at the elbow (ulnar tunnel syndrome, UTS) is the second most common and debilitating nerve compression syndrome after the median nerve entrapment at the wrist [1-3]. The prevalence of UTS is reported to be between 1.8 and $5.9 \%$ in the metropolitan population [4] while the incidence is reported around 30/100,000 personsyears [5]. The lack of diagnostic gold standard limits the utility of clinical examination through magnetic resonance imaging, ultrasonography (US), X-rays, or electromyographic measurements [6-8]. US has been regarded as a useful and effective medical imaging tool for clinical diagnosis of UTS [7, 9]. However, to date, there is no consensus; neither on the diagnostic methods nor on the pathological values of the US imaging-based measurements of the ulnar nerve [1,7-11].

The ulnar nerve ultrasound cross-sectional area (US-CSA) is a well-known parameter described in the literature to detect the morphological changes as the description of nerve entrapment $[1,7,9,12]$. It is reported that ulnar nerve US-CSA is increased in patients with ulnar nerve pathology, particularly in the entrapment $[2,13,14]$.

Jacob et al. [15] has reported a mean ulnar nerve US-CSA of $7.9 \mathrm{~mm}^{2}\left[7.5-8.3 \mathrm{~mm}^{2}\right]$ at the medial epicondyle for healthy patients. However, some studies use a threshold value of 10 $\mathrm{mm}^{2}$ for pathological value $[7,15]$. Yoon et al. [12] considered a measurement ratio between proximal and distal ulnar nerve US-CSA $>2.8$ as pathological. Thus, there is still a lack of consensus in the literature to assess the UTS diagnosis and monitoring [16]. Furthermore, there is an unmet need considering the ulnar nerve vascularization as increased intra and epineural vascularization could be a sign to detect UTS. Ghanei et al. [17] showed the interest to detect ulnar nerve vascularization as a high specificity in UTS.

Despite the importance of US-CSA metric, few studies evaluated the inter-observer ulnar nerve US-CSA reliability $[18,19]$.

Thus, the objective of this study was (1) to describe US-CSA in asymptomatic ulnar nerves and (2) to evaluate the interobserver reliability of these measurements assessed by two independent ultrasonographers.

\section{METHODS}

\section{Study Population}

Fifty healthy volunteers participated in this study. This study was approved by the regional ethical committee and each volunteer signed the informed consent form. The ethic committee that approved this study was CPP Ouest 2 Angers: Dossier 2017/38. Reference number: 2017-A01360-53. The study was conducted in accordance with the Declaration of Helsinki and its later amendments. The rheumatology department recruited healthy individuals 
between 18 and 70 years of age, with no history of musculoskeletal pathologies, and who were willing to volunteer for a sonographic evaluation. Exclusion criteria were upper limb musculoskeletal pathologies such as subluxation of the ulnar nerve, tendonitis, and ulnar tunnel syndrome. Among volunteers, no one suffered from any disease such as the Charcot-Marie-Tooth syndrome, which is likely to increase the ulnar nerve US-CSA. [12] Demographic and medical history data were collected including age, sex, weight, height, dominant side, use of medical antecedent (if any), and occupation. We studied 50 consecutive healthy individuals seen at the rheumatology department of the Brest teaching hospital (France) between September 2017 and February 2018.

\section{Ultrasound Evaluation}

All of the US evaluation was performed by two ultrasonographers: an expert having 20 years of US experience and an intermediate user having 2 years of US experience. For each volunteer, the ulnar nerve was scanned using greyscale and power Doppler (PD) with 2 MyLab $^{\circledR}$ Eight US systems $\left(\right.$ ESAOTE $^{\circledR}$, Genoa, Italy) resulting in an image dataset of 100 ulnar nerves. PD settings were as follows: repetition frequency was set at $750 \mathrm{~Hz}$ and color gain was set just below the level at which color noise appeared (no flow visualized at the body bone surface). The flow was assessed in two planes and confirmed by pulse wave Doppler to exclude artefacts. Low wall filters were used. After assessing greyscale images, PD was performed to detect vascularization in the longitudinal and transverse scan.

\section{Ultrasound Procedure}

The same procedure was realized twice for each patient in different rooms with two identical ultrasound devices on the same day. Each volunteer was placed in a lateral recumbent position to adequately expose elbows for image acquisition. US images were acquired at three levels: at $2 \mathrm{~cm}$ proximal to the epicondyle, at the level of the epicondyle, and at $2 \mathrm{~cm}$ distal to the epicondyle $[7,20]$. For each ulnar nerve, a longitudinal and transverse scan in greyscale and PD was performed to assess, respectively, the echostructure and surface of the nerve, and the vascularization.

\section{Statistical Analysis}

The mean ulnar nerve US-CSA, the standard deviation (SD), and the minimal and the maximal values were extracted. Mean ulnar nerve US-CSA values were compared between the dominant and non-dominant side using Student's paired $t$ test. Mean ulnar nerve US-CSA values between the male and female population were compared using Student's $t$ test. Mean ulnar nerve US-CSA were compared between them in the entire cohort at the three different locations levels using a one-way ANOVA with Tukey post hoc test. The sample size calculation agreed with recent work [18] with $n>50$ ulnar nerves to analyze reliability. The statistical power was $80 \%$ for a population of 43 patients (86 ulnar nerves). The intraclass correlation coefficient (ICC) was calculated on the ulnar nerve US-CSA measured by both physicians and individually on each level of the US image acquisition. The interobserver correlation was evaluated by the Bland and Altman method and the ICC was computed with a risk $(\alpha)$ set to 0.05 . ICC values were categorized into poor (ICC <

$0.5)$, moderate $(0.5<$ ICC $<0.75)$, good $(0.75<$ ICC $<0.90)$, and excellent (ICC $>$ 0.90). [21, 22]. The statistical analysis was carried out with MedCalc Statistical Software version 19.4.1 (MedCalc Software bv, Ostend, Belgium; https://www.medcalc.org) with the level of significance set at $p \leq 0.05$.

\section{RESULTS}

Fifty healthy volunteers were included. Demographic description of these volunteers is reported in Table 1. US-CSA measure was not significantly different between dominant and non-dominant side at any of the three levels of scanning location (Table 2). However, significant differences were found between males and females $2 \mathrm{~cm}$ proximal to the epicondyle and $2 \mathrm{~cm}$ distal to the epicondyle. The mean ulnar 
Table 1 Characteristic description of the population

\begin{tabular}{ll}
\hline Number of subjects & 50 \\
Subjects $<40$ years & 32 \\
Subjects $\geq 40$ years & 18 \\
Subjects $<24$ BMI & 35 \\
Subjects $\geq 25$ BMI & 15 \\
Number of ulnar nerves analyzed & 100 \\
Sex ratio F/M & $26 / 24$ \\
Mean age $(y e a r s) \pm$ SD [min-max] & $36 \pm 11.44$ \\
& {$[19-67]$} \\
Mean BMI $\left(\mathrm{kg} / \mathrm{m}^{2}\right) \pm \mathrm{SD}$ & $23 \pm 3.71[18-33]$ \\
$\quad[\min -\mathrm{max}]$ & \\
\hline
\end{tabular}

nerve US-CSA measured $2 \mathrm{~cm}$ proximal to the epicondyle was not statistically different than at the level of the epicondyle $(p=0.899)$. Even if no hourglass syndrome was clearly visualized with US, we found that the mean ulnar nerve US-CSA measured $2 \mathrm{~cm}$ proximal to the epicondyle was statistically different than $2 \mathrm{~cm}$ distal to the epicondyle (swelling ratio 1.12, $p=0.031$ ). Besides, the mean ulnar nerve USCSA measured at the level of the epicondyle was statistically different than $2 \mathrm{~cm}$ distal to the epicondyle (swelling ratio $1.14, p=0.045$ ). As per the available measurement techniques in the MyLab ${ }^{\circledR}$ Eight US system, the calculation of the ulnar nerve US-CSA was obtained with no decimal point and as a whole number (Fig. 1). The highest prevalence of ulnar nerves whose ulnar nerve US-CSA was greater than $8 \mathrm{~mm}^{2}$ and $10 \mathrm{~mm}^{2}$ was observed at the level of the epicondyle in $24 \%$ and $7 \%$ of our healthy population, respectively (Fig. 2). The lowest prevalence of ulnar nerves whose ulnar nerve US-CSA was greater than $8 \mathrm{~mm}^{2}$ and $10 \mathrm{~mm}^{2}$ was observed at the level of $2 \mathrm{~cm}$ distal to the epicondyle in $7 \%$ and $0 \%$ of our population, respectively (Fig. 2).

The reliability results revealed the ICC of $0.79,0.75$, and 0.57 at proximal, epicondyle, and distal levels, respectively. These results were expressed with the Bland and Altman method
(Fig. 3). Concerning the ulnar nerve vascularization, we found no Doppler signal around or inside the nerve whatever the scanning technique in the longitudinal or transverse scan.

\section{DISCUSSION}

Accurate clinical diagnosis of UTS in adults is an under-researched area with a lack of clinical guidelines. Clinicians and surgeons need imaging modalities to assess UTS for diagnosing, monitoring, and follow-up. Ultrasonography is an accessible tool with emerging interest. However, there is still an unmet need concerning the cut-off values for establishing the ulnar nerve US-CSA for UTS detection. This study reported ulnar nerve US-CSA measurement in a healthy adult population and proposed guidelines and cut-off value(s) for future clinical classification of ulnar nerve US-CSA. US imaging was used to acquire ulnar nerve images at three locations and the procedure adapted to quantify US-CSA was reported to have excellent inter-observer reliability. We found that ulnar nerve US-CSA measured at both levels $\left(7.07 \pm 1.95 \mathrm{~mm}^{2}\right.$ at $2 \mathrm{~cm}$ proximal to the epicondyle, and $7.17 \pm 2.11 \mathrm{~mm}^{2}$ at the level of the epicondyle) were more important than at $2 \mathrm{~cm}$ distal to the epicondyle $\left(6.29 \pm 1.54 \mathrm{~mm}^{2}\right.$; $p=0.031$ and $p=0.045$; respectively). In consequence, there was a proximal to distal gradient in the measurement of the ulnar nerve CSA when following it in its ulnar tunnel course. At the distal level, ulnar nerve US-CSA interagreement ICC was moderate (0.57) suggesting that the location of the measurement was more difficult to assess for non-expert sonographers. However, we reported the same significant differences considering the ulnar nerve US-CSA at the proximal versus distal level measurement and at the level of epicondyle $(p=0.02)$ versus distal level measurement $(p=0.04)$ for the expert sonographer. We found no ulnar nerve vascularization using PD at any levels. The epineural and intraneural vascularization would not have been detected in our study, which could be explained by the Doppler sensitivity of the machine and the frequency of the probe. 


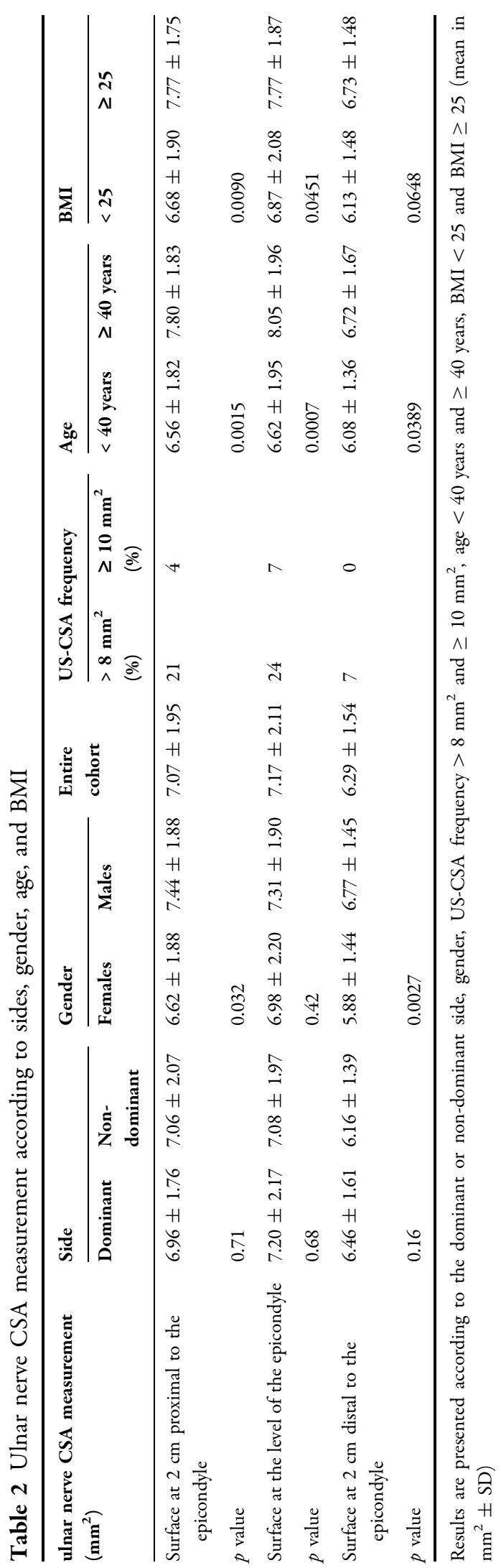

Results of the current study found no difference between the dominant and non-dominant side in a healthy cohort, whereas differences were found in gender at proximal and distal epicondylar levels. This study revealed that men had an increased ulnar nerve measurement at the proximal and distal level compared to women. On the contrary, at the level of the epicondyle, no significant differences were observed. These results were in accordance Jacob et al. [15] who reported similar observations. On the contrary, Jacob et al. [15] reported no differences between right and left sides (not based on handedness). Yalcin et al. [20] reported no significant difference between the dominant and non-dominant side.

Our study reported the mean ulnar nerve USCSA values of $7.07 \pm 1.95 \mathrm{~mm}^{2}$ at $2 \mathrm{~cm}$ proximal to the epicondyle, $7.17 \pm 2.11 \mathrm{~mm}^{2}$ at the level of the epicondyle and $6.29 \pm 1.54 \mathrm{~mm}^{2}$ at $2 \mathrm{~cm}$ distal to the epicondyle. Jacob et al. [15] reported a mean ulnar nerve US-CSA value at the epicondyle level of $7.9 \pm 3.1 \mathrm{~mm}^{2}$ on a healthy cohort of 200 volunteers (123 females) whereas Wiesler et al. [7] reported a mean ulnar nerve US-CSA of $6.5 \pm 1 \mathrm{~mm}^{2}$ on a healthy cohort of 30 volunteers (19 females) and showed no significant measures differences at three different levels of measurement. In comparison, we found differences in measurement and this could be attributed to the different scanning protocol and measurement position for Jacob et al. [15] and Wiesler et al. [7]. Another explanation could be the location of the ulnar nerve measurement. Jacob et al. [15] measured ulnar nerve US-CSA only at the epicondylar level. Wiesler et al. [7] studied ulnar nerve US-CSA at three levels similar to the current study and found that the mean ulnar nerve CSA in healthy volunteers was $6.5 \mathrm{~mm}^{2}$ (range, 5-10; SD $1 \mathrm{~mm}^{2}$ ) at the ulnar tunnel level. The last issue could be the difference between populations. According to Jacob et al. [15], age could be a factor influencing ulnar nerve USCSA values with an increase in ulnar nerve USCSA after 40 years of age. Our study included volunteers with the age range of 19-67 years old and we showed similar results with ulnar nerve US-CSA statistically increased in 


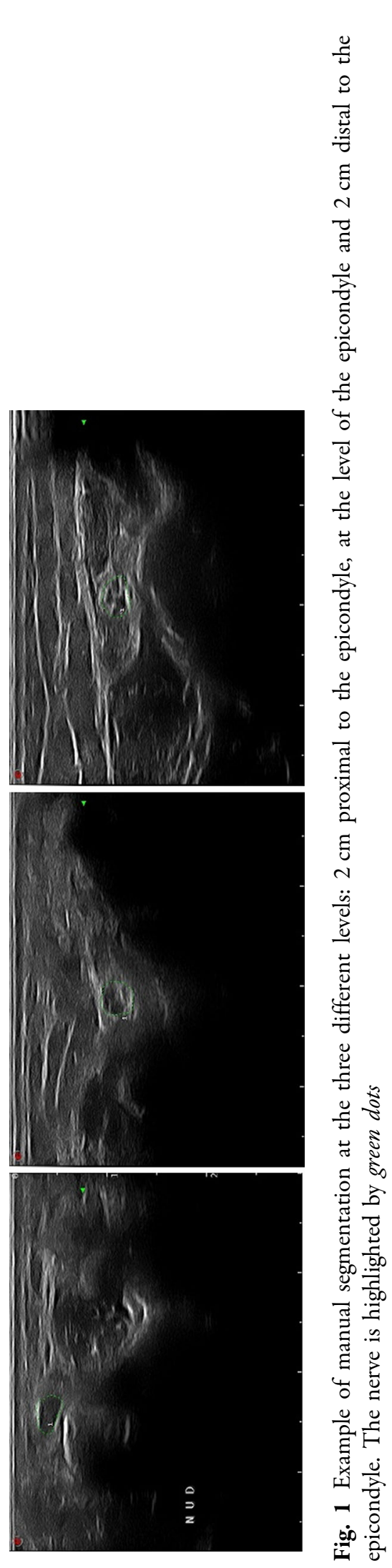

patients $\geq 40$ years compared to those $<40$ years (Table 2 ).

Compared to ulnar nerve US-CSA values of the ulnar nerve in a healthy cohort, Yalcin et al. [20] found a US-CSA normal ulnar nerve value between 5.5 and $5.7 \mathrm{~mm}^{2}$ at the three levels of measurement and Weisler found an ulnar nerve US-CSA values at $6.5 \mathrm{~mm}^{2}$ at the epicondyle. Both authors compared asymptomatic and symptomatic ulnar nerve and found that measurement of the ulnar nerve US-CSA was higher for the symptomatic side. A cut-off value of $10 \mathrm{~mm}^{2}$ or higher for CSA yields a sensitivity of $93 \%(14 / 15$ elbows) and a specificity of $98 \%$ (59/60 elbows). The positive and negative predictive values were 93\% (14/15 elbows) and 98\%, respectively (59/60 elbows).

Regarding the cut-off values to consider a pathological ulnar nerve CSA, Chiou et al. [11] found a cut-off of $7.5 \mathrm{~mm}^{2}$. However, in their study, they did not use the conventional automatic US surface setting for measuring the ulnar nerve. Jacob et al. [15] reported normal values of mean ulnar nerve US-CSA of $7.9 \mathrm{~mm}^{2}$ [7.5-8.3 $\left.\mathrm{mm}^{2}\right]$. Other authors advanced a threshold to a value of $10 \mathrm{~mm}^{2}[7,15]$.

Wiesler et al. reported [7] a cut-off of $10 \mathrm{~mm}^{2}$, with a specificity of $98 \%$ and sensitivity of $93 \%$, a positive predictive value of $93 \%$ and a negative predictive value of $98 \%$. The measurements were also performed according to a comparable method at the same three levels. We found similar results since, in their study on a healthy population, 4,7 , and $0 \%$ of ulnar nerves had an ulnar nerve US-CSA higher than $10 \mathrm{~mm}^{2}$, respectively, at $2 \mathrm{~cm}$ proximal to the epicondyle, at the level of the epicondyle and at $2 \mathrm{~cm}$ distal to the epicondyle. If we take into account our results of ulnar nerve cut-off values, we could estimate that using a cut-off value of $8 \mathrm{~mm}^{2}$ will lead to a sensitivity of 79 , 76 , and $93 \%$ of the healthy population at proximal, epicondylar, and distal levels. However, with a cut-off value set at $10 \mathrm{~mm}^{2}$, we would achieve the sensitivity of 96, 93, and $100 \%$ at proximal, epicondylar, and distal levels. This is in accordance with recent literature. Ulnar nerve CSA of healthy participants, at different levels locations, rarely exceeded $10 \mathrm{~mm}^{2}$. In consequence, authors considered 


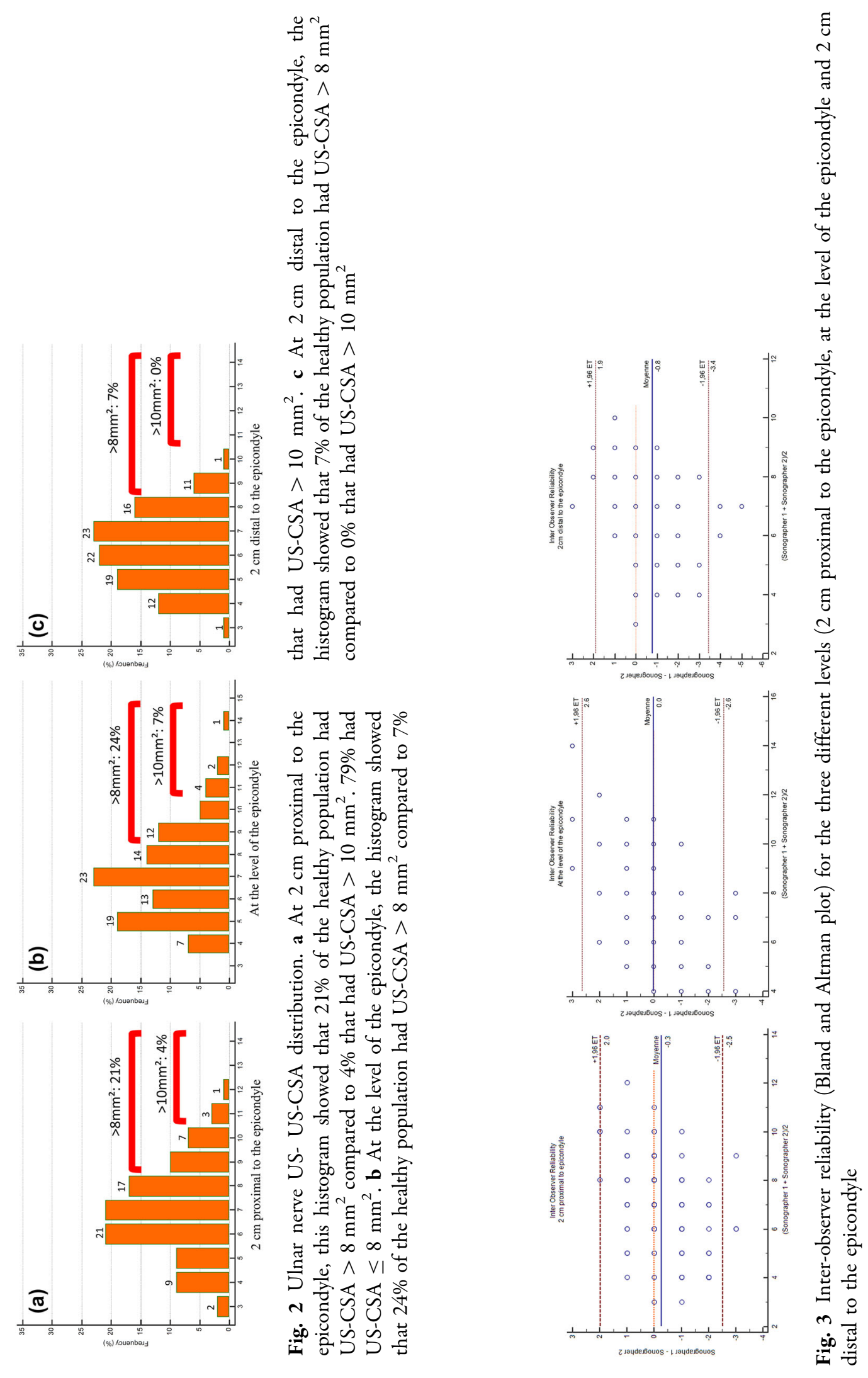


this value as a cut-off point [23]. In most of the relevant literature, the inter-observer reliability of the US-CSA measurement has not been reported. In our study, we performed a reliability exercise between two ultrasonographers on healthy controls and showed that the inter-observer reliability for measuring ulnar nerve USCSA was good whatever the operator's level of experience (either expert or novice) at $2 \mathrm{~cm}$ proximal to the epicondyle and at the level of the epicondyle while it was moderate at $2 \mathrm{~cm}$ distal to the epicondyle. In previous studies, the US inter-reliability was studied by Thoirs et al. in 2005 on ten healthy subjects and obtained a good result: the Pearson's co-efficient was 0.56 with no statistical difference between sonographers $p=0.70$ [19]. A recent study [18] also evaluated inter and intra-observer reliabilities in measuring the ulnar nerve US-CSA at the level of the medial epicondyle and obtained good results in intra-observer reliability and moderate results for inter-observer reliability.

The main limitation of our study was that our population was from a single center. The size of the population sample studied is one of the highlights of this study with 100 healthy ulnar nerves.

\section{CONCLUSIONS}

US ulnar nerve measurement surface at the elbow in a healthy population is a simple, dynamic, and reliable technique at two levels locations $(2 \mathrm{~cm}$ proximal to the epicondyle and at the epicondyle). A cut-off value of $>10 \mathrm{~mm}^{2}$ seemed to be a good value to detect UTS in case of suspicion of symptomatic clinical findings. This is the first step towards normal US B-mode ulnar nerve measurements at the elbow to further detect pathological US findings as UTS.

\section{ACKNOWLEDGEMENTS}

Funding. This study was funded by CHRU of Brest. No Rapid Service Fee was received by the journal for the publication of this article.
Authorship. All named authors meet the International Committee of Medical Journal Editors (ICMJE) criteria for authorship for this article, take responsibility for the integrity of the work as a whole, and have given their approval for this version to be published.

Disclosures. Hoel Letissier, Guillaume Dardenne, Alain Saraux, Dominique Le Nen, Bhushan Borotikar, and Sandrine Jousse-Joulin have nothing to disclose.

Compliance with ethics guidelines. This study was approved by the regional ethical committee and each volunteer signed the informed consent form. The ethic committee that approved this study was CPP Ouest 2 Angers: Dossier 2017/38. Reference number: 2017-A01360-53. The study was conducted in accordance with the Declaration of Helsinki and its later amendments.

Data availability. The datasets generated during and/or analyzed during the current study are available from the corresponding author on reasonable request.

Open Access. This article is licensed under a Creative Commons Attribution-NonCommercial 4.0 International License, which permits any non-commercial use, sharing, adaptation, distribution and reproduction in any medium or format, as long as you give appropriate credit to the original author(s) and the source, provide a link to the Creative Commons licence, and indicate if changes were made. The images or other third party material in this article are included in the article's Creative Commons licence, unless indicated otherwise in a credit line to the material. If material is not included in the article's Creative Commons licence and your intended use is not permitted by statutory regulation or exceeds the permitted use, you will need to obtain permission directly from the copyright holder. To view a copy of this licence, visit http://creativecommons.org/licenses/by$\mathrm{nc} / 4.0 /$. 


\section{REFERENCES}

1. Yoon JS, Kim BJ, Kim SJ, Kim JM, Sim KH, Hong SJ, et al. Ultrasonographic measurements in cubital tunnel syndrome. Muscle Nerve. 2007;36:853-5. https://doi.org/10.1002/mus.20864.

2. Mondelli M, Filippou G, Frediani B, Aretini A. Ultrasonography in ulnar neuropathy at the elbow: relationships to clinical and electrophysiological findings. Neurophysiologie Clinique $=$ Clinical Neurophysiology. 2008;38:217-26. https://doi.org/ 10.1016/j.neucli.2008.05.002.

3. Robertson C, Saratsiotis J. A review of compressive ulnar neuropathy at the elbow. J Manipulative Physiol Ther. 2005;28:345. https://doi.org/10.1016/ j.jmpt.2005.04.005.

4. An T, Evanoff B, Boyer M, Osei D. The prevalence of cubital tunnel syndrome: a cross-sectional study in a U.S. Metropolitan Cohort. J Bone Jt Surg. 2017;99: 408-16. https://doi.org/10.2106/JBJS.15.01162.

5. Osei DA, Groves AP, Bommarito K, Ray WZ. Cubital tunnel syndrome: incidence and demographics in a national administrative database. Neurosurgery. 2017;80:417-20. https://doi.org/10.1093/neuros/ nyw061.

6. Kitzinger HB, Aszmann OC, Moser VL, Frey M. Significance of electroneurographic parameters in the diagnosis of chronic neuropathy of the ulnar nerve at the elbow. Handchir Mikrochir Plast Chir. 2005;37:276-81. https://doi.org/10.1055/s-2005865861.

7. Wiesler ER, Chloros GD, Cartwright MS, Shin HW, Walker FO. Ultrasound in the diagnosis of ulnar neuropathy at the cubital tunnel. J Hand Surg. 2006;31:1088-93. https://doi.org/10.1016/j.jhsa. 2006.06.007.

8. Beekman R, Schoemaker MC, Van Der Plas JP, Van Den Berg LH, Franssen H, Wokke JH, et al. Diagnostic value of high-resolution sonography in ulnar neuropathy at the elbow. Neurology. 2004;62: 767-73. https://doi.org/10.1212/01.wnl. 0000113733.62689.0d.

9. Nakamichi K, Tachibana S. Ultrasonographic measurement of median nerve cross-sectional area in idiopathic carpal tunnel syndrome: diagnostic accuracy. Muscle Nerve. 2002;26:798-803. https:// doi.org/10.1002/mus.10276.

10. Bayrak AO, Bayrak IK, Turker H, Elmali M, Nural MS. Ultrasonography in patients with ulnar neuropathy at the elbow: comparison of cross-sectional area and swelling ratio with electrophysiological severity. Muscle Nerve. 2010;41:661-6. https://doi. org/10.1002/mus.21563.

11. Chiou HJ, Chou YH, Cheng SP, Hsu CC, Chan RC, Tiu CM, et al. Cubital tunnel syndrome: diagnosis by high-resolution ultrasonography. J Ultrasound Med. 1998;17:643-8.

12. Yoon JS, Walker FO, Cartwright MS. Ultrasonographic swelling ratio in the diagnosis of ulnar neuropathy at the elbow. Muscle Nerve. 2008;38: 1231-5. https://doi.org/10.1002/mus.21094.

13. Gruber H, Glodny B, Peer S. The validity of ultrasonographic assessment in cubital tunnel syndrome: the value of a cubital-to-humeral nerve area ratio (CHR) combined with morphologic features. Ultrasound Med Biol. 2010;36:376-82. https://doi. org/10.1016/j.ultrasmedbio.2009.09.008.

14. Ozturk E, Sonmez G, Colak A, Sildiroglu HO, Mutlu $\mathrm{H}$, Senol MG, et al. Sonographic appearances of the normal ulnar nerve in the cubital tunnel. J Clin Ultrasound. 2008;36:325-9. https://doi.org/10. 1002/jcu.20486.

15. Jacob D, Creteur V, Courthaliac C, Bargoin R, Sassus $\mathrm{B}$, Bacq $\mathrm{C}$, et al. Sonoanatomy of the ulnar nerve in the cubital tunnel: a multicentre study by the GEL. Eur Radiol. 2004;14:1770-3. https://doi.org/10. 1007/s00330-004-2401-6.

16. Andrews K, Rowland A, Pranjal A, Ebraheim N. Cubital tunnel syndrome: Anatomy, clinical presentation, and management. J Orthopaed. 2018;15: 832-6. https://doi.org/10.1016/j.jor.2018.08.010.

17. Ghanei ME, Karami M, Zarezadeh A, Sarrami AH. Usefulness of combination of grey-scale and color Doppler ultrasound findings in the diagnosis of ulnar nerve entrapment syndrome. J Res Med Sci. 2015;20:342-5.

18. Fink A, Teggeler M, Schmitz M, Janssen J, Pisters M. Reproducibility of ultrasonographic measurements of the ulnar nerve at the cubital tunnel. Ultrasound Med Biol. 2017;43:439-44. https://doi.org/10.1016/ j.ultrasmedbio.2016.09.022.

19. Thoirs K, Williams M, Wilkinson M. Sonographic measurements of the ulnar nerve and the cubital tunnel at the elbow: interobserver reproducibility. Radiography. 2005;11:277-83. https://doi.org/10. 1016/j.radi.2005.01.007.

20. Yalcin E, Unlu E, Akyuz M, Karaahmet OZ. Ultrasound diagnosis of ulnar neuropathy: comparison of symptomatic and asymptomatic nerve thickness. J Hand Surg Eur. 2014;39:167-71. https://doi.org/ $10.1177 / 1753193413484627$. 
21. Fleiss JL. Reliability of measurement. In: The design and analysis of clinical experiments. New York: Wiley; 1999. p. 1-32.

22. Koo TK, Li MY. A guideline of selecting and reporting intraclass correlation coefficients for reliability research. J Chiropractic Med. 2016;15:155. https://doi.org/10.1016/j.jcm.2016.02.012.
23. Chang KV, Wu WT, Han DS, Ozcakar L. Ulnar nerve cross-sectional area for the diagnosis of cubital tunnel syndrome: a meta-analysis of ultrasonographic measurements. Arch Phys Med Rehabil. 2017. https://doi.org/10.1016/j.apmr.2017.08.467. 